\title{
Potencial de produtividade da mandioca em função da época de plantio em ambiente subtropical
}

\author{
Yield potential of Cassava Crop in a function of planting date in a subtropical environment \\ Josias Moreira Borges*, Alencar Junior Zanon, Michel Rocha da silva, Darlan Scapini Balest, \\ Alexandre Ferigolo Alves, Charles Patrick de Oliveira de Freitas, Vanderlei Both, Amanda Thirza Lima \\ Santos
}

Universidade Federal de Santa Maria, Santa Maria, RS, Brasil. *Autor para correspondência: josiasdosul@gmail.com

Submissão: 10/07/2019 / Aceite: 22/06/2020

\begin{abstract}
RESUMO
Estudos de potencial e lacuna de produtividade são de extrema importância a nível mundial, para identificar e diminuir as lacunas de produtividade. O objetivo deste estudo foi estimar o potencial produtivo através do modelo Simanihot e quantificar a lacuna de produtividade na cultura da mandioca para dois locais do Rio Grande do Sul. Foram utilizados para as simulações os municípios de Santa Maria (cultivo anual) e São Luiz Gonzaga (cultivo anual e bianual). As datas de plantio foram definidas desde primeiro de agosto de 2017 até primeiro de maio de 2018. O melhor potencial de produtividade encontrado para a cultura da mandioca neste estudo foi $64,6 \mathrm{Mg} \mathrm{ha}^{-1}, 50,2 \mathrm{Mg} \mathrm{ha}^{-1}$ de raízes e 47,3 Mg ha $\mathrm{Mg}^{-1}, 38,9 \mathrm{Mg} \mathrm{ha}^{-1}$ de parte aérea, respectivamente para São Luiz Gonzaga e para Santa Maria. Os plantios a partir do zoneamento de risco climático, apresentam uma lacuna de 0,364 $\mathrm{Mg} \mathrm{ha}^{-1}$ em São Luiz Gonzaga e 0,282 $\mathrm{Mg} \mathrm{ha}^{-1}$ em Santa Maria a cada dia de atraso. Estes resultados apontam que a antecipação do plantio para o primeiro decêndio de agosto expressa as maiores produtividades e diminuem as lacunas para ambos os locais.
\end{abstract}

PALAVRAS-CHAVE: Manihot esculenta Crantz, zoneamento agrícola, segurança alimentar.

\begin{abstract}
Studies of potential and yield potential are extremely important worldwide, to identify and narrow yield potential. The objective of this study was to simulate cassava yield potential and water-limited yield potential using the Simanihot model. Cassava yield gap and the planting date effect were estimated for two locations in Rio Grande do Sul, Brazil (Santa Maria and São Luiz Gonzaga). Due to weather conditions, in Santa Maria cassava cycle was defined as annual and São Luiz Gonzaga as annual and biannual. Planting dates were set from August $1^{\text {st }} 2017$ to May $1^{\text {st }} 2018$, and harvested on Jun $15^{\text {th }} 2018$. Results showed that cassava express higher yield potential on early planting (August $10^{\text {th }}$ ), achieving 64.6 $\mathrm{Mg} \mathrm{ha}^{-1}$ and $50.2 \mathrm{Mg} \mathrm{ha}^{-1}$ of tuber roots and 47.3 $\mathrm{Mg} \mathrm{ha}^{-1}$ and $38.9 \mathrm{Mg} \mathrm{ha}^{-1}$ of aboveground biomass, in São Luiz Gonzaga and Santa Maria, respectively. Planting dates after the beginning of the climatic risk zoning, present a yield reduction of $0.364 \mathrm{Mg} \mathrm{ha}^{-1}$ in São Luiz Gonzaga and $0.282 \mathrm{Mg} \mathrm{ha}^{-1}$ in Santa Maria, for each day of delay on the planting date. These results indicate that planting cassava during the first ten days of August expresses the highest yields and reduces the gaps for both locations.
\end{abstract}

KEYWORDS: Manihot esculenta Crantz, climate risk zoning, food security.

\section{INTRODUÇÃO}

A mandioca (Manihot esculenta Crantz) é uma cultura de subsistência, característica das propriedades familiares em países tropicais em desenvolvimento, como na África Subsaariana, que tem nessa cultura a principal fonte de energia (ALEXANDRATOS \& BRUINSMA 2012). A Organização das Nações Unidas (ONU) elegeu a mandioca como o alimento do século XXI (ALLEM 1994, HOWELER et al. 2013). Além do importante papel das raízes, as folhas também podem ser utilizadas como fonte proteica na alimentação, pois, apresentam em média 17,9\%, de proteína (MOTA et al. 2011, MOREIRA et al. 2017, TAGLIAPIETRA et al. 2019).

O Brasil é o quarto produtor mundial de mandioca com 20,1 milhões de toneladas de raízes (FAO 
2019), sendo cultivada principalmente em pequenas propriedades, formando a base alimentar das populações de baixa renda (TIRONI et al. 2017). A produtividade média nos últimos 10 anos é baixa com 14,6 Mg ha ${ }^{-1}$. O Rio Grande do Sul, sétimo produtor nacional, com 4,5\% da produção do Brasil em 2019 , apresenta produtividade média de $17,8 \mathrm{Mg} \mathrm{ha}^{-1}$ (IBGE 2019), muito abaixo do potencial de $60 \mathrm{Mg} \mathrm{ha}^{-1}$ encontrado em experimentos na Colômbia (COCK 1990), $62 \mathrm{Mg} \mathrm{ha}^{-1}$ em Roraima - Brasil (OLIVEIRA et al. 2017) e 32,8 Mg ha ${ }^{-1}$ atingida em experimentos no Rio Grande do Sul - Brasil (TIRONI et al. 2015). Uma alternativa viável para o aumento de produção nesse cenário é o aumento da produtividade vertical de alimentos visando à redução das lacunas de produtividade, ou seja, reduzindo a diferença entre a produtividade atingível e a produtividade obtida atualmente nas lavouras comerciais (GODFRAY et al. 2010).

Estudos de potencial e lacuna de produtividade são de extrema importância a nível mundial, entre os projetos de maior envergadura a nível global, está o GLOBAL YIELD GAP ATLAS (2019), com o objetivo de identificar e diminuir as lacunas de produtividade das principais culturas agrícolas ao redor do mundo. Esses estudos são ainda mais importantes em países em desenvolvimento, como na América Latina, África e Ásia. O potencial de produtividade (Yp) é o rendimento de uma cultura quando a água e os nutrientes não limitam, e o estresse biótico é efetivamente controlado, ou seja, o Yp é definido pelos fatores determinantes como material genético, concentração de $\mathrm{CO}_{2}$, radiação solar, fotoperíodo e temperatura do ar. O potencial de produtividade limitado por água $(\mathrm{Yw})$ de lavouras de sequeiro são limitados pelos fatores de água (quantidade e distribuição) e solo.

A simulação de culturas por modelos matemáticos é uma das maneiras mais confiáveis de estimar o potencial de produtividade quando calibrados e validados dentro de um sistema de cultivo definido. $\mathrm{O}$ modelo Simanihot pode ser utilizado em estudos numéricos, como ferramenta de simulação da cultura da mandioca, já calibrado e validado para o estado do Rio Grande do Sul para cultivares de mesa, forrageira e de indústria, pois apresenta bom desempenho em simulações de crescimento, desenvolvimento e produtividade de parte área e de raízes tuberosas de mandioca (TIRONI et al. 2017). Além disso, este modelo possui uma interface gráfica que permite aos usuários selecionar um dos dois modelos de balanço hídrico do solo representando bem o teor real de água no solo (TIRONI et al. 2017).

Os fatores limitantes e redutores associados ao manejo inadequado, ocasionam menor produtividade em relação ao potencial de produtividade, estabelecendo assim, as lacunas de produtividade (yield gap) na cultura da mandioca (DE PONTI et al. 2012). Estudos indicam época adequada de plantio, irrigação para suprir o déficit hídrico, cultivares tolerantes ao déficit hídrico, doses de fertilizantes e corretivos de solo, defensivos agrícolas e adubação verde reduzem a lacuna de produtividade na cultura de mandioca no Brasil (VISSES et al. 2018).

Resultados de pesquisas são incipientes quanto a influência da época de plantio na cultura da mandioca, principalmente no Rio Grande do Sul, e alguns estudos indicam que a data de plantio afeta diretamente a disponibilidade dos elementos meteorológicos ao longo do ciclo de desenvolvimento da cultura determinando o filocrono e o número de folhas na mandioca (SCHONS et al. 2007), enquanto outros estudos inferem que o número de folhas na haste principal, por ocasião do início de acumulação de amido nas raízes tuberosas, não varia em razão da época de plantio (FAGUNDES et al. 2009). Nesse sentido, o objetivo deste estudo foi simular através do modelo Simanihot o potencial de produtividade (Yp) e o potencial de produtividade limitado por água $(Y w)$ da parte área (folhas, hastes e pecíolo) e raízes tuberosas, e quantificar a lacuna de produtividade com o atraso do plantio na cultura da mandioca para dois locais do Rio Grande do Sul no período indicado pelo zoneamento de risco climático.

\section{MATERIAL E MÉTODOS}

O potencial de produtividade (Yp e $Y w)$ e as lacunas de produtividade $(Y g)$ foram simuladas através do modelo Simanihot, um modelo matemático baseado em processos, que simula a produção de parte área e raízes de mandioca, sendo calibrado e validado para o Rio Grande do Sul, segundo metodologia descrita por TIRONI et al. (2017). O Yp e Yw foram simulados para dois locais do Rio Grande do Sul: São Luiz

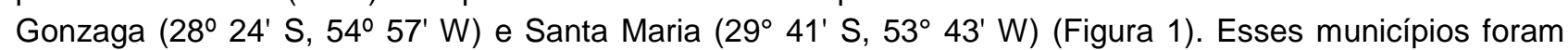
definidos por apresentarem estações meteorológicas e por representarem distintas regiões indicadas para a produção da mandioca no RS (VAN BUSSEL et al. 2015). Santa Maria é uma região apta apenas para o cultivo anual e São Luiz Gonzaga é uma região apta para o cultivo anual e bianual (MALUF et al. 2011).

Para simular o potencial de produtividade (Yp) foram inseridos dados de entrada obtidos no Banco de Dados Meteorológicos para Ensino e Pesquisa (BDMEP) do Instituto Nacional de Meteorologia (INMET), estação de São Luiz Gonzaga-A852 Código OMM: 86961 e Santa Maria-A803 Código OMM: 86977. Os 
elementos meteorológicos utilizados no modelo Simanihot foram: precipitação, temperatura máxima, temperatura mínima, umidade relativa do ar, radiação solar e velocidade do vento. Os dados diários contemplaram o período de 1 de agosto de 2017 a 15 de junho de 2018.

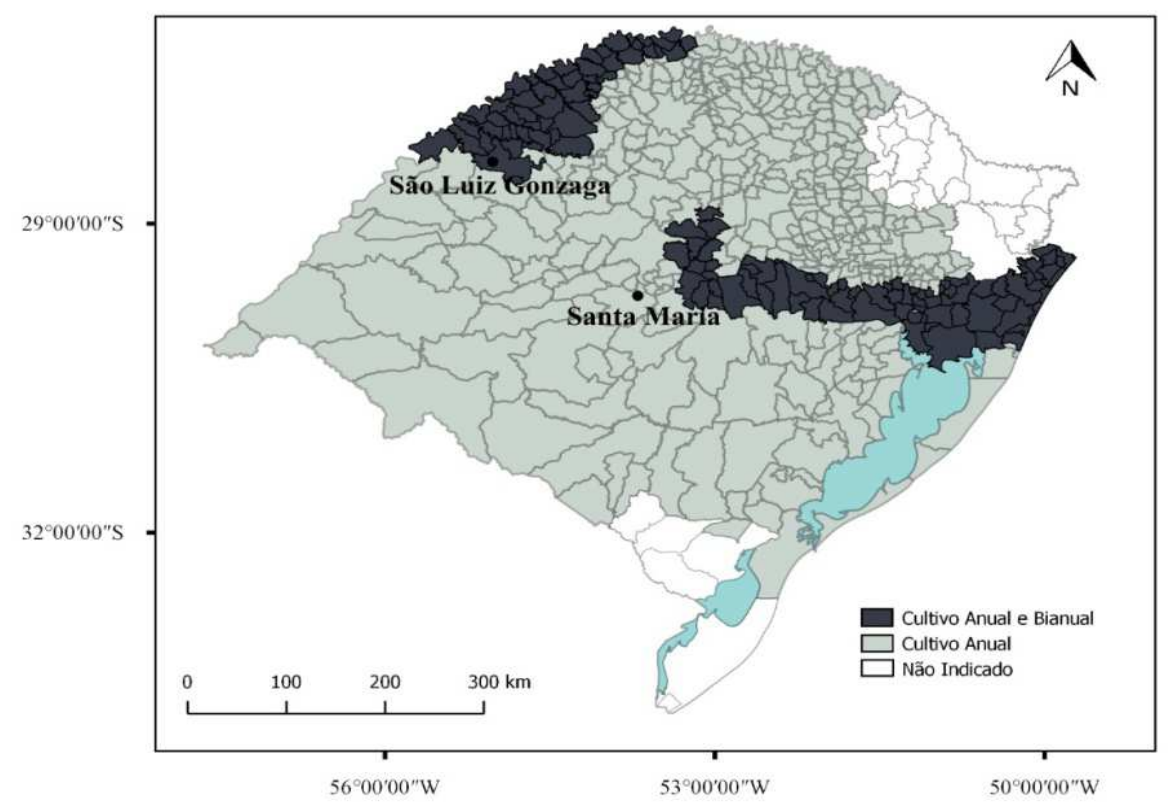

Figura 1. Mapa do estado do Rio Grande do Sul, indicando as localizações de cultivo e os pontos pretos indicam onde foram estimados os Potenciais de produtividade (MALUF et al. 2011).

Figure 1. Rio Grande do Sul State map, indicating cassava cultivation sites, being the black points indicators of where the yield potential was estimated (MALUF et al. 2011).

Para o potencial de produtividade limitado por água $(\mathrm{Yw})$ foram realizadas simulações com os mesmos dados de entrada, porém ativando o balanço hídrico de THORNTHWAITE \& MATHER (1955) (já incluso no modelo Simanihot) (GABRIEL et al. 2014). As simulações de plantio foram realizadas para os dias 1,11 e 21 , dos meses de agosto de 2017 a 1 de maio de 2018, com densidade de 12.500 plantas ha $^{-1}$ e a colheita para todas as simulações foi no dia 15 de junho de 2018. Levou-se em consideração a tendência linear dos plantios dentro do zoneamento de risco climático para quantificar a lacuna de produtividade com o atraso do plantio na cultura da mandioca para dois os locais do Rio Grande do Sul.

Foram selecionadas para estimar o potencial de produtividade da parte aérea (hastes e folhas) e raízes as cultivares FEPAGRO RS 13 por ser de aptidão forrageira com importância no aporte de silagem para animais, por possuir qualidades nutritivas e destacando-se por apresentar concentração de proteínas bruta de 37,9\% na matéria seca, e a SÃO JOSÉ de aptidão de mesa, onde que minimamente processada, representa maior valor agregado (TIRONI et al. 2017, TAGLIAPIETRA et al. 2019).

As lacunas de produtividade $\mathrm{Yg}, \mathrm{Yg} 1, \mathrm{Yg} 2$ e Yg3 foram então calculadas através da diferença entre Yp e Yw, Yan, Yae e Yam. Onde:

$\begin{array}{ll}\text { (1) } & Y g=Y p-Y w \\ (2) & Y g 1=Y p-Y a n \\ (3) & Y g 2=Y p-Y a e \\ (4) & Y g 3=Y p-Y a m\end{array}$

em que Yg, Yg1, Yg2 e Yg3 são as lacunas de produtividade; Yp é o potencial de produtividade simulada pelo modelo; Yw é a produtividade limitada por água simulada pelo modelo; Yan é a média de produtividade nacional (Brasil); Yae é a média de produtividade estadual (Yae) (Rio Grande do Sul); Yam é a média de produtividade municipal (São Luiz Gozada e Santa Maria). Yan, Yae e Yam são as médias de produtividade de lavouras de produtores, sendo dados obtidos através do IBGE (2019), safra 2018/2019.

\section{RESULTADOS E DISCUSSÃO}

O potencial de produtividade de raízes limitado por água $(Y w)$ não difere do potencial de produtividade (Yp) (Figura 2). Já o Yw da parte aérea da cultivar FEPAGRO RS 13, em Santa Maria, apresentou a lacuna de produtividade $(\mathrm{Yg})$ de $3 \mathrm{Mg} \mathrm{ha}^{-1}$ em relação ao $\mathrm{Yp}$ de parte aérea (Figura 2A). A precipitação pluviométrica acumulada (Figura 3) no período de avaliação foi de $1447 \mathrm{~mm}$ em Santa Maria e 
1650 mm em São Luiz Gonzaga, suprindo a necessidade fisiológica durante o ciclo da cultura para produtividade de raízes, pois precipitações consideradas ideais para a cultura variam de $1000 \mathrm{~mm}$ a 2000 mm anuais bem distribuídos (MALUF et al. 2011).
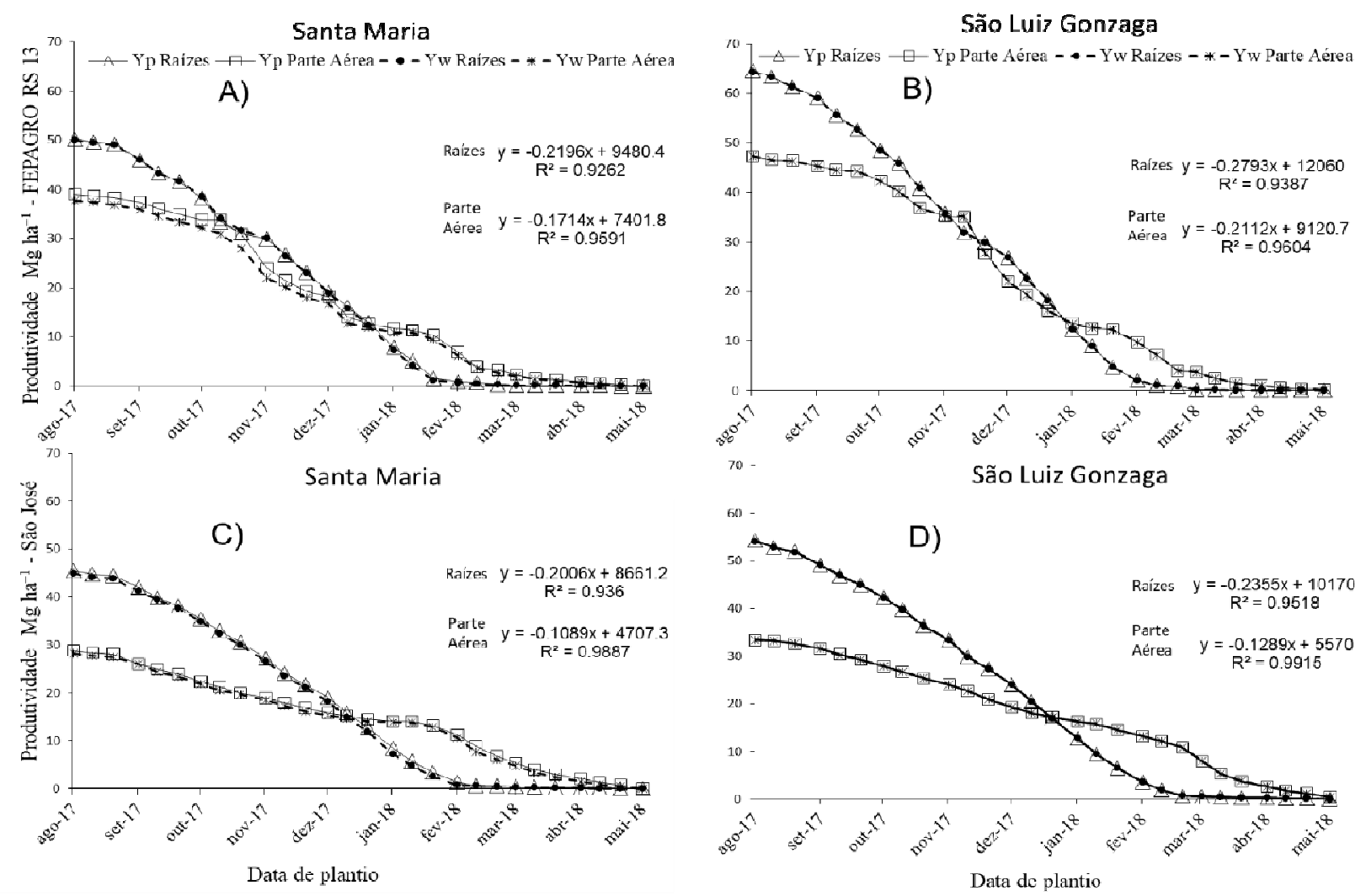

Figura 2. Relação entre data de plantio, potencial de produtividade (Yp) e potencial de produtividade limitado por água $(Y w)$ da parte aérea e raízes de mandioca, para as cultivares FEPAGRO RS 13 (A e B) e SÃO JOSÉ (C e D) nos municípios de Santa Maria e São Luiz Gonzaga, RS. As equações de linha de tendência (A, B, C e D) referem-se ao potencial produtivo (Yp) das Raízes e da Parte Aérea.

Figure 2. Relationship between planting time, yield potential (Yp) and water-limited yield potential (Yw) of cassava aboveground and roots biomass for FEPAGRO RS 13 (A and B) and SÃO JOSÉ (C and D) cassava cultivars in Santa Maria, RS (Left) and São Luiz Gonzaga, RS (Right). The trend line equations refer to $Y p$ of the roots and aboveground area $(A, B, C$, and $D)$.

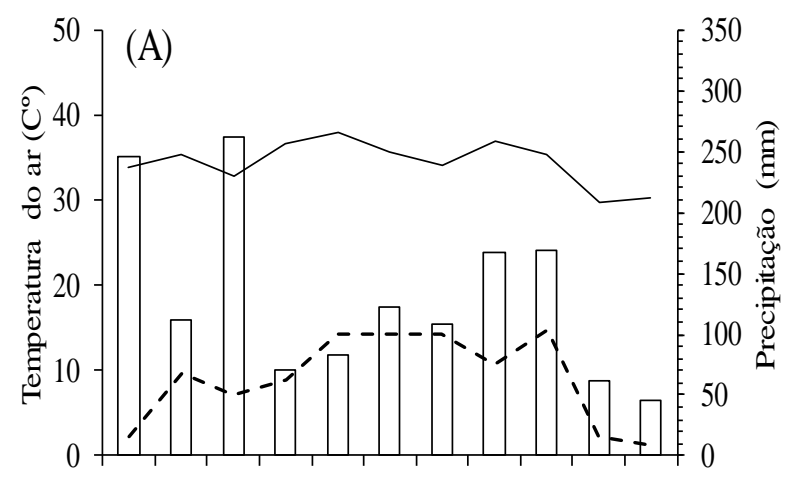

ago set out nov dez jan fev mar abr mai jun Meses

$\square$ Precipitação acumulada - - - Tmin — Tmax

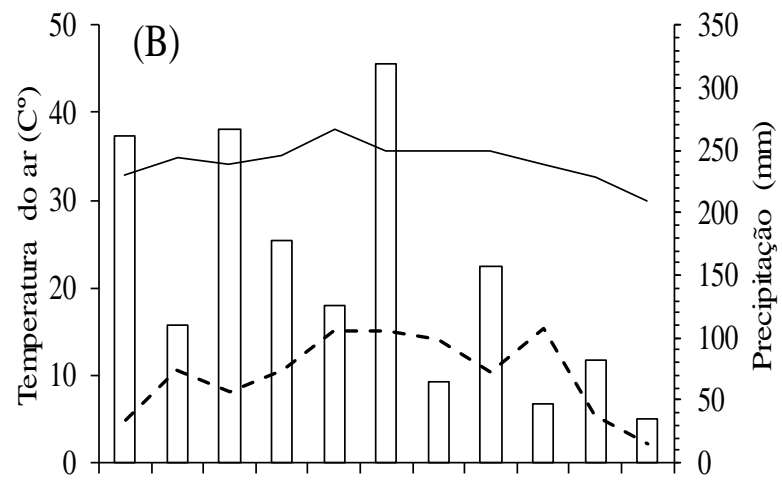

ago set out nov dez jan fev mar abr mai jun Meses

$\square$ Precipitação acumulada - - - Tmin — Tmax

Figura 3. Precipitação mensal acumulada $(\mathrm{mm})$ e temperaturas máximas e mínimas do ar em Santa Maria, RS (A) e São Luiz Gonzaga, RS (B), de agosto de 2017 a junho de 2018.

Figure 3. Cumulative monthly precipitation $(\mathrm{mm})$ and maximum and minimum air temperature in Santa Maria, RS (A) and São Luiz Gonzaga, RS (B), from August 2017 to June 2018. 
Para a parte aérea da cultivar FEPAGRO RS 13 existe uma lacuna de produtividade $(\mathrm{Yg})$ de $3 \mathrm{Mg}$ $\mathrm{ha}^{-1} \mathrm{em}$ Santa Maria (Figura 2A), mostrando que há sensibilidade hídrica na produtividade da parte aérea. Isso ocorre devido à resposta maior da parte vegetativa da planta a disponibilidade de água (TIRONI et al. 2019). Nesse sentido, o modelo Simanihot interpreta as propriedades físicas dos solos; número de camadas e profundidade máxima das raízes. Sendo assim, o solo de Santa Maria, classificado como um Argissolo, apresenta menor armazenamento de água por conter menor profundidade comparado aos Latossolos encontrados em São Luiz Gonzaga (STRECK et al. 2008). De maneira geral, considerando as raízes, o modelo não expressou deficiência hídrica, pois ocorreu boa distribuição da precipitação pluviométrica no RS (Figura 3). Estudos fisiológicos afirmam que a cultura da mandioca tolera secas prolongadas, pois ocorre na planta fechamento parcial dos estômatos permitindo que o potencial hídrico das folhas permaneça estável, não comprometendo a fotossíntese e maximizando a eficiência no uso da água (MORGANTE et al. 2020). Logo, considerando as raízes (Figura 4), apesar da água ser um fator limitante do potencial de produtividade, não houve diferença na lacuna de produtividade $(Y g)$ entre $Y p$ e $Y w$ para ambos locais e cultivares.

A)

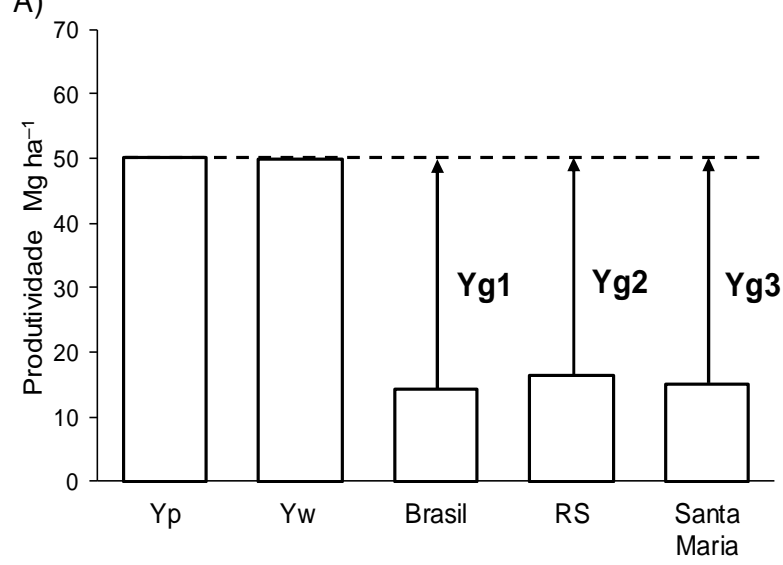

B)

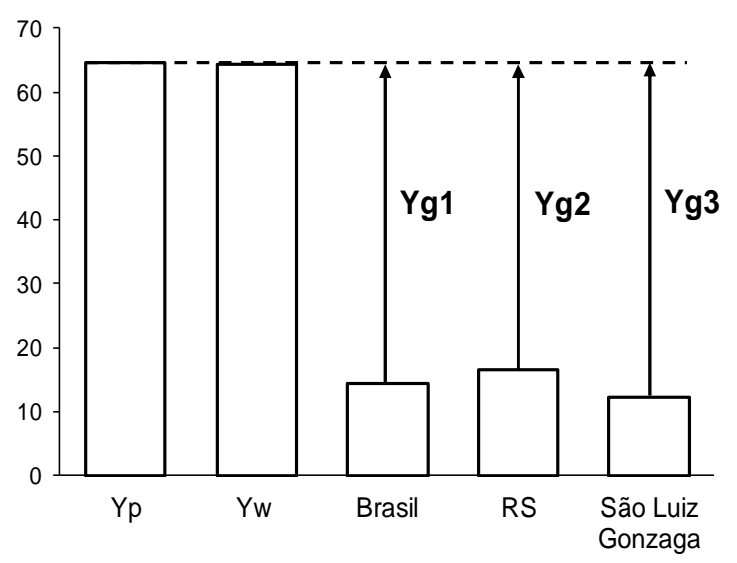

Figura 4. Potencial de produtividade de raízes tuberosas de mandioca (Yp); potencial de produtividade de raízes tuberosas limitado por água $(Y w)$, estimados pelo modelo Simanihot e as Lacunas de produtividade $(\mathrm{Yg})$ no município de Santa Maria (A) e São Luiz Gonzaga (B); e média de produtividade dos produtores de mandioca safra 2018/2019 segundo Instituto Brasileiro de Geografia e Estatística (IBGE) do Brasil e Rio Grande do Sul (RS).

Figure 4. Yield potential (Yp), water-limited yield potential (Yw), estimated by the Simanihot model and Yield Gap (Yg) in Santa Maria (A) and São Luiz Gonzaga (B); Average cassava yield in 2018/2019 from Instituto Brasileiro de Geografia e Estatística (IBGE) of Brazil and Rio Grande do Sul (RS).

As maiores produtividades de raízes ocorreram quando o plantio foi no início de agosto diminuindo à medida que se atrasa o plantio (Figura 2). Este fato ocorre devido à mandioca ser sensível a temperatura e ao fotoperíodo, sendo que a cultura da mandioca sob temperaturas altas e fotoperíodo longo diminui a proporção de matéria seca que chega às raízes (ALVES 2006). Além disso, a data de plantio influencia a disponibilidade dos elementos meteorológicos ao longo do ciclo de desenvolvimento, pois a temperatura e a radiação solar são alteradas ao longo do ano. Logo, esses fatores determinam o Yp, sendo responsáveis pela inclinação da curva (Figura 2).

Plantios realizados entre 1 de agosto e 10 de setembro resultam em melhores produtividades de raízes. Vale salientar que para a região com cultivo bianual, a data de plantio indicada pelo zoneamento de risco climático se estende de 21 de agosto a 30 de novembro, e para a de cultivo anual, 11 de setembro a 30 de novembro (MALUF et al. 2011). Levando em consideração a tendência linear dos plantios dentro do zoneamento de risco climático, o potencial de produtividade é penalizado em até $0,364 \mathrm{Mg} \mathrm{ha}^{-1} \mathrm{dia}^{-1}$ em São Luiz Gonzaga ( $y=15488-0,364 x)$ e 0,282 $\mathrm{Mg} \mathrm{ha}^{-1} \mathrm{dia}^{-1}$ em Santa Maria ( $\mathrm{y}=12178-0,282 \mathrm{x}$ ).

Para ambas cultivares, tanto parte aérea como raízes tuberosas, nas simulações em Santa Maria (Figura 2A e 2C), o platô observado no início da época de simulação da função Yp foi mais evidenciado que em São Luiz Gonzaga. Isso foi devido a ocorrência de geada e baixas temperaturas que aumentaram o tempo da emergência em relação ao plantio. Logo, os resultados dos plantios em agosto foram mais estáveis em Santa Maria. Foram registrados no mês de agosto em Santa Maria temperaturas mínimas de 2,4 ${ }^{\circ} \mathrm{C}$ e $4,9 \stackrel{\circ}{\circ} \mathrm{C}$ em São Luiz Gonzaga, sendo que a partir do mês de agosto as temperaturas aumentam 
gradativamente (Figura 3). Temperaturas entre $10 \stackrel{\circ}{\circ} \mathrm{C}$ e $38{ }^{\circ} \mathrm{C}$ são toleráveis e inferiores a $3{ }^{\circ} \mathrm{C}$ por longo período de cultivo, tornam-se inviável a produção de mandioca (MALUF et al. 2011).

Há um período estável no Yp da parte aérea para os plantios nos meses de dezembro de 2017 e janeiro de 2018 em ambas as localidades e cultivares. As hastes da planta, a exemplo das raízes, acumulam maior massa em função do tempo, porém, por consequência do efeito da senescência das folhas até a colheita (15/06/18), os plantios entre 190 e 140 dias antes da colheita apresentam estabilidade na massa da parte aérea, pois há maior massa de folhas nos plantios ao final desse platô, do que no início. Sendo assim, o acúmulo de massa da haste compensa a senescência das folhas ocasionando o fenômeno. Frente a isso, pensando em silagem de mandioca, pode-se lograr proveito em retardar o plantio para início de fevereiro, por que tem potencial de produtividade semelhante aos plantios de dezembro e janeiro, porém haverá maior número de folhas entregues para a elaboração da silagem, possibilitando vantagem nutritiva, ainda ter um Yp de parte aérea na cultura da mandioca próximo de $12 \mathrm{Mg} \mathrm{ha}^{-1}$. Trabalhos realizados por NAPASIRTH et al. (2015), KURNIAWAN et al. (2019) e LI et al. (2019) demostram bom desempenho na utilização de silagem de mandioca na alimentação animal.

A média de produtividade de raízes dos últimos 10 anos dos produtores de mandioca no Brasil, RS, Santa Maria e São Luiz Gonzaga são, respectivamente: 14,4; 16,4; 15,0 e 12,3 $\mathrm{Mg} \mathrm{ha}^{-1}$ (IBGE 2019). Essas médias estão muito abaixo do potencial de produtividade da cultura da mandioca (Yp). Tendo em vista que o déficit hídrico definitivamente não é fator limitante no RS, logo, fatores limitantes como nutrição e fatores redutores como presença plantas daninhas, pragas e doenças, somados a outros manejos que determinam o Yp, são os responsáveis pelas lacunas (Yg1, Yg2, Yg3) observadas na Figura 4. O município de São Luiz Gonzaga expressam $19 \%$ do potencial de produtividade, $64,6 \mathrm{Mg} \mathrm{ha}^{-1}$, totalizando uma lacuna de $81 \%$ (Figura 4B). Já a média de produtividade dos produtores de Santa Maria atingiu $30 \%$ dos $50,2 \mathrm{Mg} \mathrm{ha}^{-1}$ do Yp de Santa Maria, com uma lacuna de 70\% (Figura 4A). A média de produtividade dos produtores de mandioca do Brasil e RS correspondem 22,2 e 25,5\%, respectivamente, do Yp (Figura 4B). Estudo realizado no Brasil, especificamente na região de Rio Pardo, RS, apresenta potencial produtivo de $48,3 \mathrm{Mg} \mathrm{ha}^{-1}$, valores próximos encontrados neste trabalho, e relatam que fatores como manejo, déficit hídrico, eficiência climática e agronômica causam as lacunas de produtividade (VISSES et al. 2018). Salienta-se a necessidade de estudos futuros com métodos mais detalhados, com experimentos a campo e aplicação de questionários aos produtores, para se levantar os fatores promotores das lacunas.

Outra questão importante em relação a Figura 4, é a hipótese do excesso hídrico, não percebido pelo modelo Simanihot, correlacionar-se às lacunas de produtividade apresentadas, pois há alta pluviosidade no período indicado pelo zoneamento de risco climático, por exemplo: a média dos locais investigados, obtida pelo BDMEP, dos últimos 30 anos, se aproxima de $250 \mathrm{~mm}$ para a precipitação acumulada para o mês de outubro. A cultura da mandioca quando exposta à ambientes com solos úmidos, podem proporcionar ambiente favorável a patógenos e a falta de oxigênio para as raízes (SAIRAM et al. 2008, TAIZ \& ZEIGER 2009), consequentemente, menor sobrevivência de plantas. O manejo no preparo da área deve atender a drenagem suficiente para o excesso hídrico não limitar o potencial de produtividade. Nesse sentido, pelo modelo Simanihot não ser sensível a esses fatores, a cultura carece de estudos para validar/calibrar o modelo e embasar na tomada de decisão dos produtores quanto ao excesso hídrico.

A média de produtividade de raízes dos últimos 10 anos dos produtores de mandioca no Brasil $(14,4$ $\left.\mathrm{Mg} \mathrm{ha}^{-1}\right)$, RS (16,4 Mg ha $\left.{ }^{-1}\right)$, Santa Maria (15,0 Mg ha ${ }^{-1}$ ) e São Luiz Gonzaga (12,3 Mg ha $\mathrm{Ma}^{-1}$ ) são baixas comparada ao potencial produtivo que a cultura pode expressar. Esta diferença deve-se a diversos fatores, sendo um dos principais, a data de plantio. Este tipo de trabalho é de extrema importância, pois identificou o quanto a cultura pode produzir e a lacuna de produtividade existente nas regiões de estudo. Por meio destas informações, políticas públicas podem ser direcionadas aos produtores, fomentando o cultivo e aumentado a produtividade das lavouras de mandioca.

\section{CONCLUSÃO}

A antecipação do plantio para o primeiro decêndio de agosto expressa o melhor potencial de produtividade para cultura da mandioca de $64,6 \mathrm{Mg} \mathrm{ha}^{-1}, 50,2 \mathrm{Mg} \mathrm{ha}^{-1}$ de raízes e 47,3 Mg ha-1, 38,9 Mg $\mathrm{ha}^{-1}$ de parte aérea, respectivamente, em São Luiz Gonzaga e para Santa Maria.

Os plantios a partir do zoneamento de risco climático, apresentam uma lacuna de $0,364 \mathrm{Mg} \mathrm{ha}^{-1} \mathrm{em}$ São Luiz Gonzaga e 0,282 $\mathrm{Mg} \mathrm{ha}^{-1} \mathrm{em}$ Santa Maria a cada dia de atraso.

\section{REFERÊNCIAS}

ALEXANDRATOS N \& BRUINSMA J. 2012. World agriculture towards 2030/2050: the 2012 revision. Rome: FAO. 160p. 
ALLEM AC. 1994. The origin of Manihot esculenta Crantz (Euphorbiaceae). Genetics Resources and Crop Evolution 41 : 133-150.

ALVES AAC. 2006. Fisiologia da mandioca. In: EMBRAPA Mandioca e Fruticultura Tropical. Aspectos socioeconômicos e agronômicos da mandioca. Cruz das Almas: EMBRAPA. p.138-169.

COCK JH. 1990. La yuca: nuevo potencial para un cultivo tradicional. Cali: CIAT. 240p.

DE PONTI T et al. 2012. The crop yield gap between organic and conventional agriculture. Agricultural Systems 108: 19.

FAGUNDES LK et al. 2009. Desenvolvimento vegetativo em diferentes hastes da planta de mandioca em função da época de plantio. Ciência Rural 39: 657-663.

FAO. 2019. Food and Agriculture Organization of The United Nations - Publications. Disponível em: http://www.fao.org/home/en. Acesso em: 17 mar. 2019.

GABRIEL LF et al. 2014. Simulating cassava growth and yield under potential conditions in Southern Brazil. Agronomy Journal 106: 1119-1137.

GLOBAL YIELD GAP ATLAS. 2019. Global Yield Gap and Water Productivity Atlas. Disponível em: http://www.yieldgap.org. Acesso: 17 mar. 2019.

GODFRAY HCJ et al. 2010. Food security: the challenge of feeding 9 billion people. Science 327: 812-818.

HOWELER R et al. 2013. Save and grow: Cassava. A guide to sustainable production intensification. Rome: FAO. 129p.

IBGE. 2019. Instituto Brasileiro de Geografia e Estatística. Levantamento Sistemático da Produção Agrícola - LSPA. Disponível em: https://sidra.ibge.gov.br/home/lspa/brasil. Acesso em: 15 mar. 2019.

KURNIAWAN W et al. 2019. Quality and fermentation characteristic of corn stover - rubber cassava (Manihot glaziovii M.A.) combination silage. IOP Conference Series Earth and Environmental Science 287: 012022.

LI M et al. 2019. Silage fermentation, chemical composition and ruminal degradation of king grass, cassava foliage and their mixture. Grassland Science 65: 210-215.

MALUF JRT et al. 2011. Zoneamento Agroclimático da Mandioca no Estado do Rio Grande do Sul - Uma alternativa para a produção de etanol. Porto Alegre: FEPAGRO. 60p. (Boletim Técnico 22).

MOREIRA GLP et al. 2017. Composição bromatológica de mandioca (Manihot esculenta) em função do intervalo entre podas. Revista de Ciências Agrárias 40: 144-153.

MORGANTE CV et al. 2020. Genetic and physiological analysis of early drought response in Manihot esculenta and its wild relative. Acta Physiologiae Plantarum 42: 22.

MOTA ADS et al. 2011. Perfil de fermentação e perdas na ensilagem de diferentes frações da parte aérea de quatro variedades de mandioca. Revista Brasileira de Zootecnia 40: 1466-1473.

NAPASIRTH V et al. 2015. Microbial population, chemical composition and silage fermentation of cassava residues. Animal Science Journal 86: 279-280.

OLIVEIRA NT et al. 2017. Effect of harvest time and nitrogen doses on cassava root yield and quality. Revista Brasileira de Ciências do Solo 41: e0150204.

SAIRAM RK et al. 2008. Physiology and biochemistry of waterlogging tolerance in plants. Biologia Plantarum 52: 401412.

SCHONS A et al. 2007. Emissão de folhas e início de acumulação de amido em raízes de uma variedade de mandioca em função da época de plantio. Ciência Rural 37: 1586-1592.

STRECK EV et al. 2008. Solos do Rio Grande do Sul. 2.ed. Porto Alegre: Emater. 222p.

TAGLIAPIETRA BL et al. 2019. Mandioca para alimentação humana e animal. 1.ed. Santa Maria: EMATER. 104p.

TAIZ L \& ZEIGER E. 2009. Fisiologia vegetal. 4.ed. Porto Alegre: Artmed. 848p.

TIRONI LF et al. 2015. Desempenho de cultivares de mandioca em ambiente subtropical. Bragantia 74: 58-66.

TIRONI LF et al. 2017. Simanihot: um modelo baseado em processos para simular o crescimento, desenvolvimento e produtividade da mandioca. Engenharia Agrícola 37: 471-483.

TIRONI LF et al. 2019. Ecofisiologia da Mandioca Visando Altas Produtividades. Santa Maria: Editora GR. 136p.

THORNTHWAITE CW \& MATHER JR. 1955. The water balance. Centerton: Drexel Institute of Technology. 104p. (Publications in Climatology 1)

VAN BUSSEL LG et al. 2015. From field to atlas: upscaling of location-specific yield gap estimates. Field Crops Research 177: 98-108.

VISSES FA et al. 2018. Yield gap of cassava crop as a measure of food security-an example for the main Brazilian producing regions. Food Security 10: 1191-1202. 